\title{
Temperature-averaged and total free-free Gaunt factors for $\kappa$ and Maxwellian distributions of electrons ${ }^{\star}$ (Corrigendum)
}

\author{
Miguel A. de Avillez ${ }^{1,2}$ and Dieter Breitschwerdt ${ }^{2}$ \\ 1 Department of Mathematics, University of Évora, R. Romão Ramalho 59, 7000 Évora, Portugal \\ e-mail: mavillez@galaxy.lca.uevora.pt \\ 2 Zentrum für Astronomie und Astrophysik, Technische Universität Berlin, Hardenbergstrasse 36, 10623 Berlin, Germany \\ A\&A, 580, A124 (2015), https://doi .org/10 . 1051/0004-6361/201526104
}

Key words. atomic processes - radiation mechanisms: general - ISM: general - galaxies: ISM - errata, adenda

\section{Introduction}

A missing normalization coefficient in Eqs. (11) and (12) of de Avillez \& Breitschwerdt (2015) affected the results shown in Figs. 3 and 4 and discussed in Sect. 3 of that earlier publication. Here, we present the corrected version of the equations and the corresponding results discussed in Sect. 3 and shown in Figs. 3 and 4 of that paper. In addition we provide supplementary material in the form of tables covering a larger parameter space than the one previously used. These tables are available at the CDS.

\section{Temperature-averaged and total Gaunt factor}

During the interaction of an electron with the Coulomb field of an ion of ionic state $z$ and atomic number $Z$ the amount of freefree energy emitted per unit time and unit volume is given by

$\frac{d P_{\mathrm{ff}}}{d u}=\frac{8 \pi^{2}}{h c^{3}}\left(\frac{2}{3 m_{e}}\right)^{3 / 2} e^{6} z^{2} n_{e} n_{z, z} k_{B} T N_{e}\left\langle g_{f f}\left(\gamma^{2}, u\right)\right\rangle$,

with $z$ denoting the ionic state of an ion with atomic number $Z$ and number density $n_{Z, z}, n_{e}$ is the electron number density, $h$ is the Planck constant, $c$ is the speed of light, $e$ is the electron charge in Coulombs, $m_{e}$ is the electron mass, $k_{B}$ is the Boltzmann constant, $T$ is the temperature, and $\left\langle g_{f f}\left(\gamma^{2}, u\right)\right\rangle$ is the temperature averaged Gaunt factor given by (see, e.g., de Avillez \& Breitschwerdt 2017)

$\left\langle g_{f f}\left(\gamma^{2}, u\right)\right\rangle=\frac{\left(k_{B} T\right)^{1 / 2}}{N_{e}}$

$\times \int_{0}^{+\infty} \frac{1}{(y+u)^{1 / 2}} f\left[(y+u) k_{B} T\right] g_{f f}\left(\epsilon_{i}=\frac{y+u}{\gamma^{2}}, \epsilon_{f}=\frac{y}{\gamma^{2}}\right) d y$

where $f$ is the electron distribution function, $u=h v / k_{B} T$ ( $v$ is the frequency of the emitted photon), $\gamma^{2}=z^{2} \mathrm{Ry} / k_{B} T$ (Ry is the Rydberg constant), and $N_{e}$ is a normalization coefficient

\footnotetext{
* The set of tables is only available at the CDS via anonymous ftp to cdsarc.u-strasbg. fr $(130.79 .128 .5)$ or via http://cdsarc. u-strasbg.fr/viz-bin/qcat?J/A+A/620/C1
}

defined such $\left\langle g_{f f}\left(\gamma^{2}, u\right)\right\rangle=1$ when $g_{f f}\left(\gamma^{2}, u\right)=1$. For the $\kappa$ and Maxwell-Boltzmann (MB) electron distribution functions $N_{e}$ is given by

$N_{e}= \begin{cases}\frac{2}{\sqrt{\pi k_{B} T}} \int_{0}^{+\infty} G(y+u ; \kappa) d y & \text { if } \kappa>3 / 2 \\ \frac{2 e^{-u}}{\sqrt{\pi k_{B} T}} & \text { if } \mathrm{MB},\end{cases}$

with

$G(x ; \kappa)=\frac{A_{\kappa}}{\left[1+\frac{x}{\kappa-3 / 2}\right]^{\kappa+1}}$,

and

$\int_{0}^{+\infty} G(y+u ; \kappa) d y=A_{\kappa} \frac{\kappa-3 / 2}{\kappa}\left(1+\frac{u}{\kappa-3 / 2}\right)^{-\kappa}$,

which becomes $e^{-u}$ when $\kappa \rightarrow \infty$ (Fig. 1). In these expressions

$A_{\kappa}=\frac{\Gamma(\kappa+1)}{\Gamma(\kappa-1 / 2)(\kappa-3 / 2)^{3 / 2}}$ and $\kappa>3 / 2$.

Therefore, Eq. (11) in de Avillez \& Breitschwerdt (2015) must be rewritten as

$\frac{d P_{\mathrm{ff}}}{d u}=C_{\mathrm{ff}} z^{2} n_{e} n_{z, z} T^{1 / 2}$
$\times \begin{cases}\frac{1}{\int_{0}^{+\infty} G(y+u ; k) d y} \int_{0}^{+\infty} g_{\mathrm{ff}}\left(\gamma^{2}, u\right) G(y+u ; \kappa) d y & \text { if } \kappa>3 / 2 \\ e^{-u} \int_{0}^{+\infty} g_{\mathrm{ff}}\left(\gamma^{2}, u\right) e^{-y} d y & \text { if } \mathrm{MB} .\end{cases}$

where

$C_{\mathrm{ff}}=16\left(\frac{2 \pi}{3 m_{e}}\right)^{3 / 2} \frac{e^{6} k_{B}^{1 / 2}}{h c^{3}}=1.4256 \times 10^{-27} \mathrm{erg} \mathrm{cm}^{3} \mathrm{~s}^{-1} \mathrm{~K}^{-1 / 2}$. 


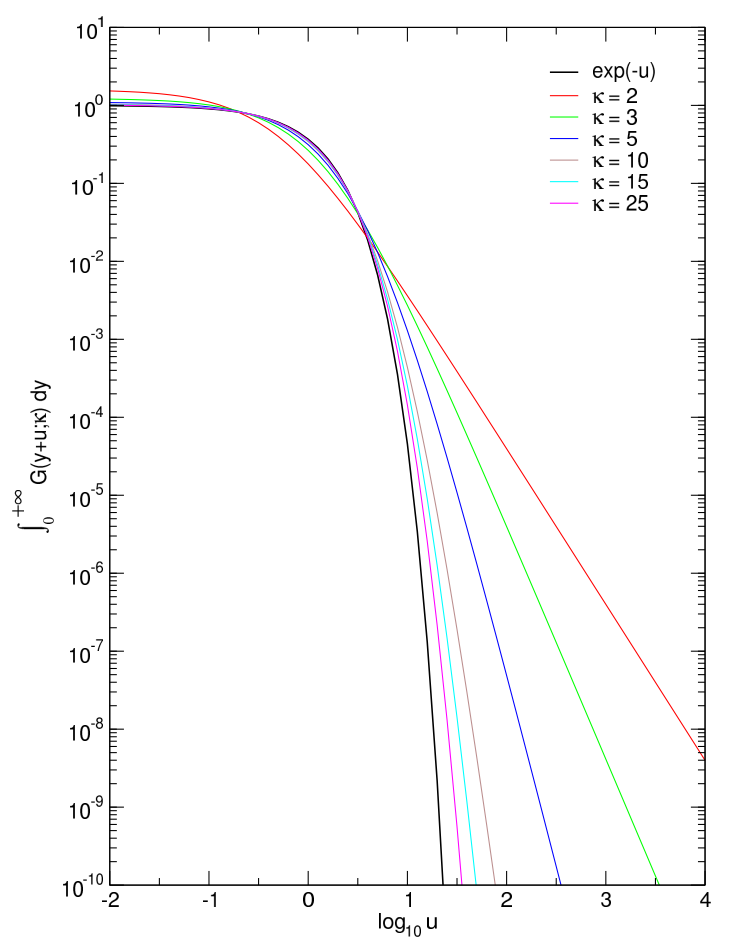

Fig. 1. Variation of Integral (5) with $\kappa$ as function of $u$. The solid black line represents $e^{-u}$ which is overlapped by the integral as $\kappa \rightarrow \infty$. by

The total free-free power associated with an ion $(Z, z)$ given

$P_{\mathrm{ff}}(T)=C_{\mathrm{ff}} z^{2} n_{e} n_{\mathrm{z}, z} T^{1 / 2} \int_{0}^{+\infty}\left\langle g_{\mathrm{ff}}\left(\gamma^{2}, u\right)\right\rangle f(u) d u$

with

$f(u)=\left\{\begin{array}{lll}\int_{0}^{+\infty} G(y+u ; \kappa) d y & \text { if } & \kappa>3 / 2 \\ e^{-u} & \text { if } & \text { MB. }\end{array}\right.$

The integral in the RHS of (8) is the total free-free Gaunt factor. When $\kappa \rightarrow \infty$ the Integral in (9) tends to $e^{-u}$ and total Gaunt factor for the MB distribution of electrons as defined by Karzas \& Latter (1961) is recovered

$g_{f f}(T)=\int_{0}^{+\infty}\left\langle g_{f f}\left(\gamma^{2}, u\right)\right\rangle e^{-u} d u$.

The top panel of Fig. 2 and the panels in Fig. 3 display the variation with $\gamma^{2}$ of the temperature-averaged Gaunt factor, $\left\langle g_{f f}\left(\gamma^{2}, u\right)\right\rangle$, for different values of $u \in\left[10^{-4}, 10^{4}\right]$ for the MB and $\kappa=2, \ldots, 1200$ distributions. The bottom panel of Fig. 2 highlights in a magnified image of the region $\gamma^{2} \in\left[10^{-2}, 10^{6}\right]$ the distribution with $\gamma^{2}$ of $\left\langle g_{f f}\left(\gamma^{2}, u\right)\right\rangle$ for different values of $u$.

It is clear that as $\kappa$ increases the temperature-averaged $\kappa$ distributed Gaunt factors approach those calculated with the MB distribution. However, the speed (with $\kappa$ variation) of this approach depends on the values of $u$. For $u<10$ this approach is faster than for $u \geq 10$ (compare top panel of Fig. 2 and the two panels in Fig. 3). For values as high as $\kappa=1200$ there is still no overlap between the $\kappa$ and Maxwellian values of $\left\langle g_{f f}\left(\gamma^{2}, u\right)\right\rangle$ for $u>10^{2}$ and $\gamma^{2}<10$ (Fig. 3). This is a consequence of the slow approach to $e^{-u}$ of the integral $\int_{0}^{+\infty} G(y+u ; \kappa) d y$ for $u \geq 10$ in comparison to $u<10$ (Fig. 1).
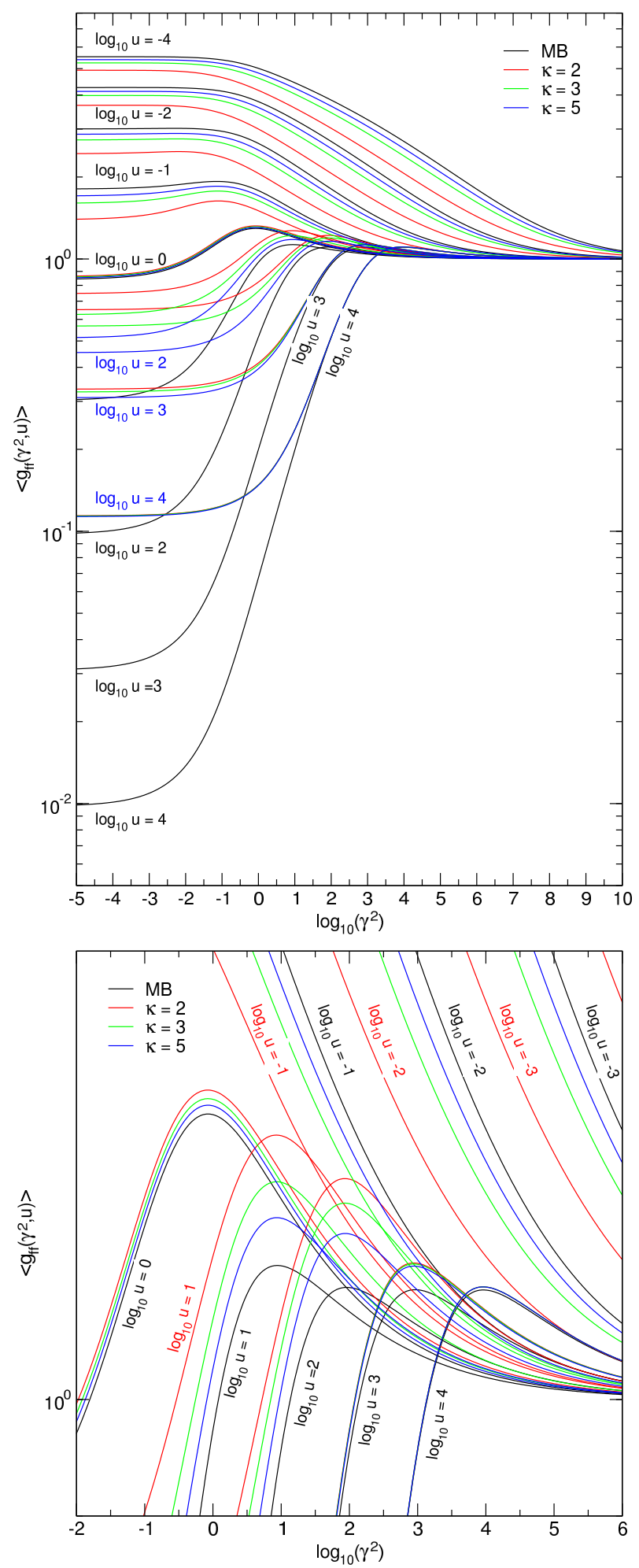

Fig. 2. Temperature-averaged Gaunt factors calculated for $\kappa=2,3,5$ and MB (black line in both panels) distributions of electrons for the range $10^{-5} \leq \gamma^{2} \leq 10^{10}$ (top panel) and a magnification of the region $\gamma^{2} \in\left[10^{-2}, 10^{6}\right]$ and $\left\langle g_{f f}\left(\gamma^{2}, u\right)\right\rangle \in[0.9,1.5]$ (bottom panel).

Figure 4 displays the total free-free Gaunt factor, $\left\langle g_{f f}\left(\gamma^{2}\right)\right\rangle$, calculated for the $\mathrm{MB}$ and $\kappa=2,3,5,10,15$, and 25 distributions. For larger $\kappa$ the $\left\langle g_{f f}\left(\gamma^{2}\right)\right\rangle$ almost overlap with the Maxwellian value as shown in the magnification of the regions $\left\langle g_{f f}\left(\gamma^{2}\right)\right\rangle \in[-1.0,0.8]$ and $\left\langle g_{f f}\left(\gamma^{2}\right)\right\rangle \in[1.38,1.45]$ (top panel 
M. A. de Avillez and D. Breitschwerdt: Temperature averaged free-free Gaunt factors for Kappa-distributions
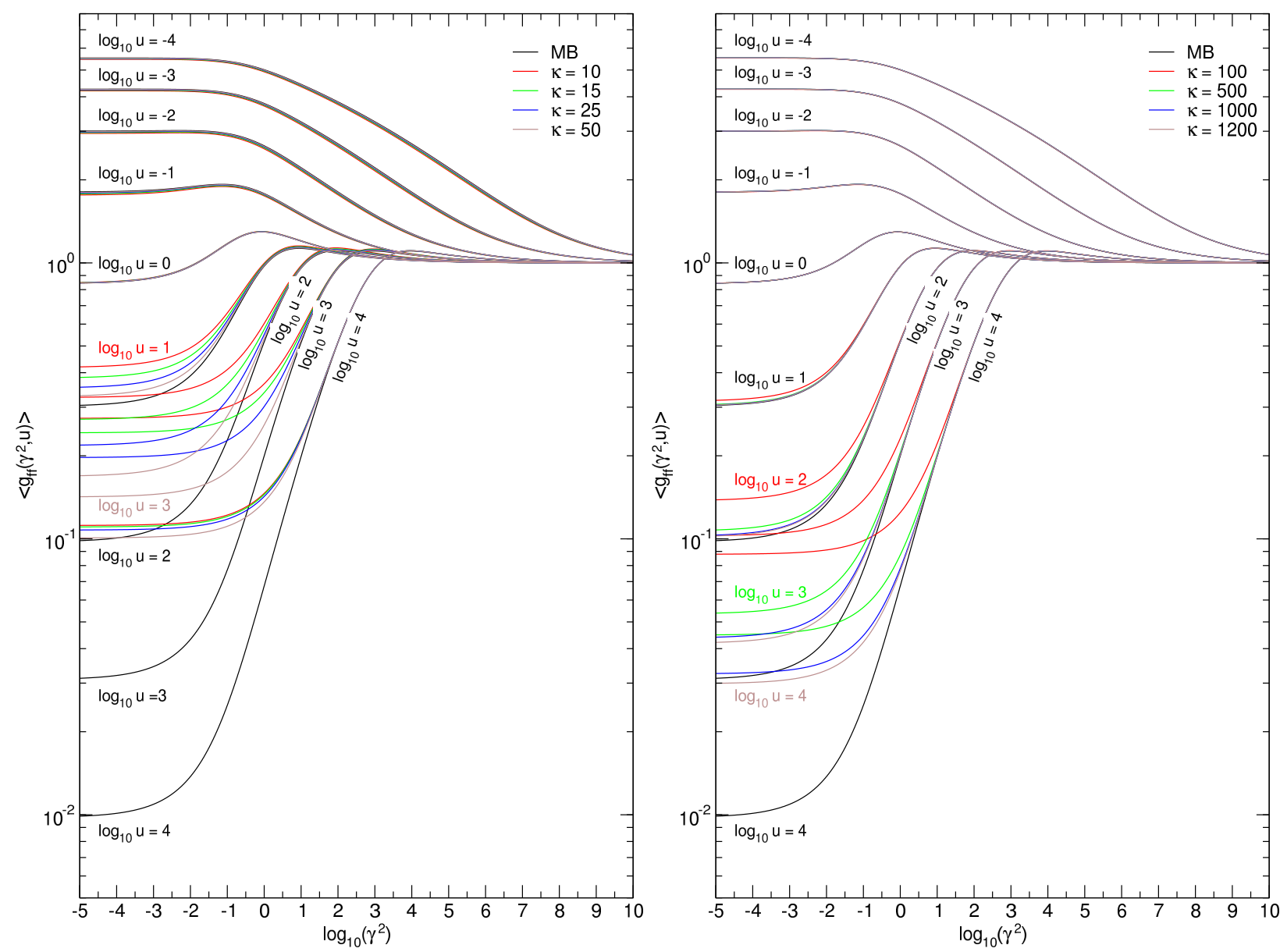

Fig. 3. Temperature-averaged Gaunt factors calculated for $\kappa=10,15,25$, and 50 (left panel), 100, 500, 1000, and 1200 (right panel) and MB (black line in both panels) distributions of electrons for the range $10^{-5} \leq \gamma^{2} \leq 10^{10}$. We note the slow approach $\left\langle g_{f f}\left(\gamma^{2}, u\right)\right\rangle$ to the Maxwellian values for $u>10$ and $\gamma^{2}<10$ for large values of the $\kappa$ parameter.

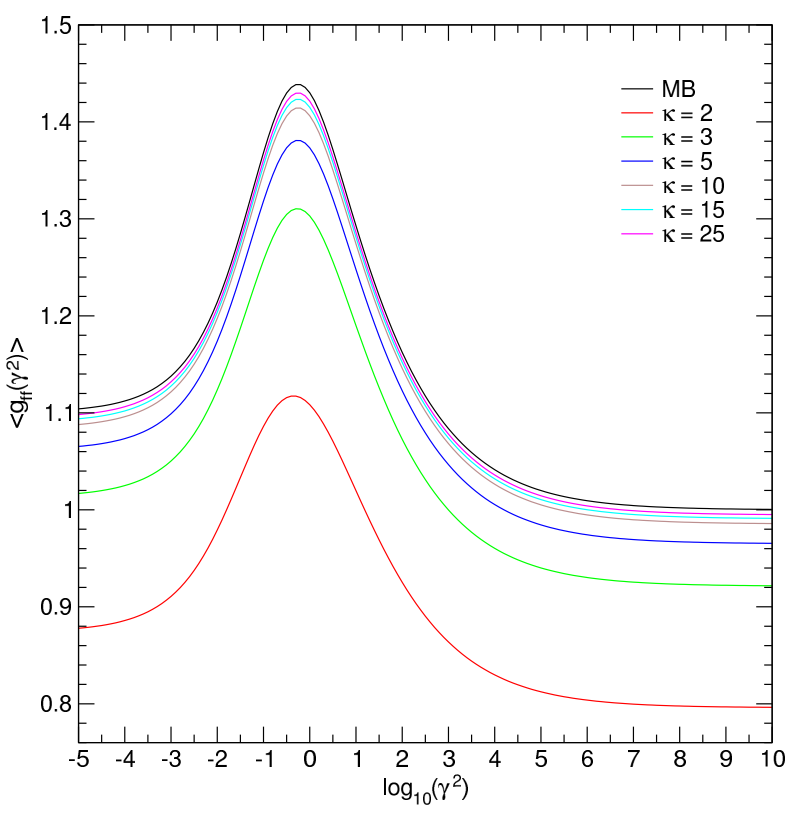

Fig. 4. Total free-free Gaunt factor calculated for $\kappa(2,3,5,10,15$, and 25) and MB distributions.

of Fig. 5), and $\left\langle g_{f f}\left(\gamma^{2}\right)\right\rangle \in[6,10]$ and $\left\langle g_{f f}\left(\gamma^{2}\right)\right\rangle \in[0.995,1.005]$ (bottom panel of same Figure). It turns out that even for $\kappa$ param-
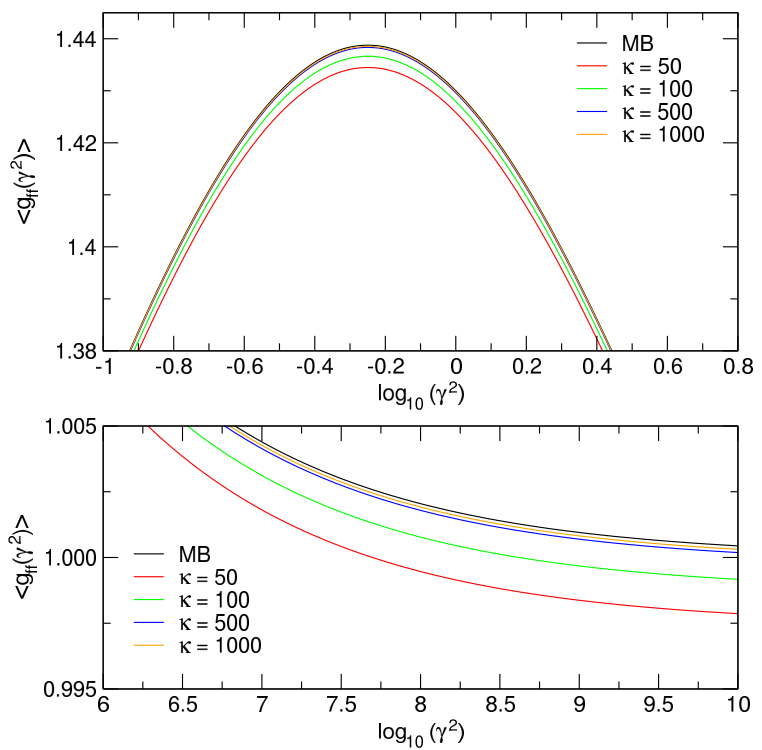

Fig. 5. Magnification of the total free-free Gaunt factor in two regions in $\gamma^{2}$ and in the $\left\langle g_{f f}\left(\gamma^{2}\right)\right\rangle$ profile shown in Fig. 4 but for $\kappa=50,100,500$, and 1000. The solid black line refers to the Maxwellian value.

eters as high as 1000 there is still a slight difference between $\left\langle g_{f f}\left(\gamma^{2}\right)\right\rangle$ calculated for the $\kappa$ and MB distributions. 


\section{Tables}

Supplementary material is available at the CDS with a set of tables referring to the temperature-averaged Gaunt factor versus $\gamma^{2}$ and different $u$, and a table for the total Gaunt factor versus $\gamma^{2}$ for results obtained with the $\kappa=$ 2, 3, 5, 10, 15, 25, 50, 100, and 500 and $\mathrm{MB}$ electron distributions. The parameter space comprises $\gamma^{2} \in\left[10^{-5}, 10^{10}\right]$ and $u \in\left[10^{-12}, 10^{11}\right]$.
Acknowledgements. This research was supported by the project Enabling Green E-science for the SKA Research Infrastructure (ENGAGE SKA), reference POCI01-0145-FEDER-022217, funded by COMPETE 2020 \& FCT, Portugal.

\section{References}

de Avillez, M. A. \& Breitschwerdt, D. 2015, A\&A, 580, A124 de Avillez, M. A. \& Breitschwerdt, D. 2017, ApJS, 232, 12 Karzas, W. J. \& Latter, R. 1961, ApJS, 6, 167 